\title{
Über Fermente in Bambusschößlingen.
}

\author{
Von \\ Kan Kato. \\ (Aus dem medizinisch-chemischen Institut der Universität zu Kyoto.) \\ (Der Redaktion zugegangen am 6. Oktober 1911.)
}

Nachdem durch die Untersuchungen von Y. Kosai ${ }^{1}$ ) und von Ginzaburo Totani ${ }^{2}$ ) dargetan worden war, daß in Bambusschößlingen die Abbauprodukte der Eiweißstoffe und die Purinbasen in nachweisbarer Menge vorhanden sind, und ferner mancherlei Beobachtungen außer Zweifel gestellt hatten, daß im pflanzlichen Stoffwechsel Fermente eine ebenso große Rolle spielen wie im tierischen, erschien es von Interesse, die Fragen zu verfolgen: ob die oben erwähnten Produkte ihre Entstehung der fermentativen Spaltung ihrer Muttersubstanzen, der Eiweißstoffe und der Nucleinstoffe, verdanken, ob außer den proteolytischen Fermenten und der Nuclease noch irgend ein Ferment in Bambusschößlingen nachzuweisen ist? Zur Entscheidung dieser Fragen sind die folgenden Versuche angestellt worden.

\section{Nuclease.}

Die Versuche, welche von Béchamp, Schützenberger, Salkowski, Hahn und Geret u. a. angestellt wurden, führten zur Erkenntnis, daß in Hefezellen ein nucleinsäurespaltendes Ferment sicher in wirksamem Zustande existiert. Leonid I wan off ${ }^{3}$ ) entdeckte dann in Schimmelpilzen ein Ferment, welches die Thymusnucleinsäure in ihre Komponenten abzubauen vermag, und bezeichnete es als Nuclease. Aus den Unter-

1) Y. Kosai, Bulletin of imperial college of agriculture and dendrology Komaba, Tokyo, 1890, No. 7, S. 37-46.

2) G. Totani, Diese Zeitschrift, Bd. 62, S. 113.

$\left.{ }^{3}\right)$ L. Iwan off, Diese Zeitschrift, Bd. 39, S. 31. 
suchungen über die Spaltung der Hefenucleinsäure durch Bakterien von A. Schittenhelm und F. Schroetter ${ }^{1}$ ) ergaben sich auch eine Reihe von Tatsachen, welche auf die Existenz der Nuclease in Bakterien hinweisen. Von Wichtigkeit bleibt endlich noch hervorzuheben, daß es Tamau Kikkoji ${ }^{2}$ ) gelang, die Nuclease aus einem in Japan einheimischen Hutpilze, Cortinellus edodes, darzustellen und ihre Wirkung genau zu ermitteln.

Bei den Versuchen, über welche im folgenden berichtet wird, kam der Preßsaft aus Bambusschößlingen zur Verwendung, der auf die folgende Weise hergestellt wurde:

$7 \mathrm{~kg}$ der von der Rinde befreiten, fein zerschnittenen Bambusschößlinge wurden in ein starkes baumwollenes Tuch eingeschlagen, auf die Preßplatte gelegt; mit einem vielfach durchlochten Preßkorb aus verzinntem Stahlblech umgeben und darauf unter starkem Druck in einer Handpresse ausgepreßt. Der so bereitete, durch Filtrierpapier filtrierte Preßsaft stellte eine dünne Flüssigkeit von gelber Farbe und von einem eigentümlichen Geruch und Geschmack nach Bambusschößlingen dar. Die Reaktion war neutral.

\section{Versuch 1.}

Probe a 1. 0,2 $\mathrm{g}$ thymusnucleinsaures Natron wurden in $10 \mathrm{ccm}$ heißem Wasser gelöst, nach dem Erkalten mit $10 \mathrm{ccm}$ Preßsaft durchgemischt und unter Zusatz von $1 \mathrm{ccm}$ Toluol bei Bruttemperatur digeriert.

Nach 24 stündiger Digestion wurde die Lösung zur Koagulation der darin vorhandenen Eiweißstoffe mit geringer Menge von Essigsäure und Kochsalz gekocht, nach dem Erkalten durch Ersetzen des verdampften Wassers auf $20 \mathrm{ccm}$ gebracht und dann filtriert. Aus $10 \mathrm{ccm}$ dieses Filtrates wurde die Phosphorsäure in der bekannten Weise durch Zusatz von Magnesiamischung als $\mathrm{MgNH}_{4} \mathrm{PO}_{4}$ niedergeschlagen und als $\mathrm{Mg}_{2} \mathrm{P}_{2} \mathrm{O}_{7}$ gewogen. Auf die ganze Probe umgerechnet wurden gefunden: $\mathrm{O}_{\mathrm{n}} 0209 \mathrm{~g} \mathrm{Mg}_{2} \mathrm{P}_{2} \mathrm{O}_{7}=0,0133 \mathrm{~g} \mathrm{P}_{2} \mathrm{O}_{5}$.

1) A. Schittenhelm u. F. Schroetter, Diese Zeitschrift, Bd. 39, S. 203. Bd. 40, S. 62 u. S. 70. Bd. 41, S. 284 .

2) T. Kikkoji, Diese Zeitschrift, Bd. 51, S. 201. 
Probe a2. (Kontrollprobe.) 0,2 g thymusnucleinsaures Natron in $10 \mathrm{ccm}$ Wasser gelöst $+10 \mathrm{ccm}$ gekochter Preßsaft. Die Mischung wurde bei Gegenwart von Toluol 24 Stunden lang bei Bruttemperatur digeriert und dann auf Phosphorsäure verarbeitet. Es wurden gefunden: $0,0041 \mathrm{~g} \mathrm{Mg}_{2} \mathrm{P}_{2} \mathrm{O}_{7}=0,0026 \mathrm{~g} \mathrm{P}_{2} \mathrm{O}_{5}$.

Probe b1. 0,2 g thymusnucleinsaures Natron in $10 \mathrm{ccm}$ Wasser gelöst $+10 \mathrm{ccm}$ Preßsaft $+1 \mathrm{ccm}$ Toluol. Die Mischung wurde 24 Stunden lang im Brutraum digeriert und auf die oben beschriebene Weise auf Phosphorsäure verarbeitet. Es wurden gefunden: $0,0213 \mathrm{~g} \mathrm{Mg}_{2} \mathrm{P}_{2} \mathrm{O}_{7}=0,0136 \mathrm{~g}_{2} \mathrm{O}_{5}$.

Probe b2. (Kontrollprobe.) 0,2 $\mathrm{g}$ thymusnucleinsaures Natron in $10 \mathrm{ccm}$ Wasser gelöst $+10 \mathrm{ccm}$ gekochter Preßsaft $+1 \mathrm{ccm}$ Toluol. Nach 24 stündiger Digestion wurde die Mischung auf Phosphorsäure verarbeitet. Es wurden gefunden: $0,0053 \mathrm{~g} \mathrm{Mg}_{2} \mathrm{P}_{2} \mathrm{O}_{7}=0,0034 \mathrm{~g} \mathrm{P}_{2} \mathrm{O}_{5}$.

Probe $\mathrm{c} 1.0,2 \mathrm{~g}$ thymusnucleinsaures Natron in $10 \mathrm{ccm}$ Wasser gelöst $+10 \mathrm{ccm}$ Preßsaft $+1 \mathrm{ccm}$ Toluol. Nach 51 stündiger Digestion wurde die Mischung auf Phosphorsäure verarbeitet. Es wurden gefunden: $0,0395 \mathrm{~g} \mathrm{Mg}_{2} \mathrm{P}_{2} \mathrm{O}_{7}=0,0252 \mathrm{~g} \mathrm{P}_{8} \mathrm{O}_{5}$.

Probe c2. (Kontrollprobe.) 0,2 g thymusnucleinsaures Natron in $10 \mathrm{ccm}$ Wasser gelöst $+10 \mathrm{ccm}$ gekochter Preßsaft $+1 \mathrm{ccm}$ Toluol. Nach 51 stündiger Digestion wurden aus der Mischung erhalten: 0,0064 $\mathrm{g} \mathrm{Mg}_{2} \mathrm{P}_{2} \mathrm{O}_{7}=0,0041 \mathrm{~g} \mathrm{P}_{2} \mathrm{O}_{5}$.

Die Sterilität der digerierten Proben war durch Ausstriche auf einem festen Nährboden und Impfung in schwach alkalische Bouillonlösung festgestellt.

Der bequemeren Übersicht halber sind die Resultate in folgender Tabelle zusammengestellt.

Die Versuchsergebnisse zeigen unzweideutig, daß bei der Digestion des thymusnucleinsauren Natrons mit dem Preßsafte aus Bambusschößlingen Phosphorsäure in reichlicher Menge entsteht. Die geringe Menge der Phosphorsäure, welche in den Kontrollproben gefunden wurde, rührt sicher von den im Preßsafte präformierten Phosphaten her. 
Tabelle 1.

\begin{tabular}{|c|c|c|c|c|c|}
\hline $\begin{array}{c}\text { Nummer } \\
\text { der } \\
\text { Proben }\end{array}$ & $\begin{array}{l}\text { Dauer } \\
\text { der Di- } \\
\text { gestion } \\
\text { in Std. }\end{array}$ & $\begin{array}{c}\text { Menge des } \\
\text { verwendeten } \\
\text { nucleinsauren } \\
\text { Natrons } \\
\text { in } \mathbf{g}\end{array}$ & $\begin{array}{c}\text { Menge } \\
\text { des ver- } \\
\text { wendeten } \\
\text { Preßsaftes } \\
\text { in ccm }\end{array}$ & $\begin{array}{c}\text { Menge } \\
\text { der ge- } \\
\text { fundenen } \\
\mathrm{P}_{2} \mathrm{O}_{5} \\
\text { in } \mathrm{g}\end{array}$ & Bemerkungen \\
\hline a1 & 24 & 0,2 & 10 & 0,0133 & $\begin{array}{l}\text { mit dem frischen } \\
\text { Preßsaft versetzt }\end{array}$ \\
\hline a2 & 24 & 0,2 & 10 & 0,0026 & $\begin{array}{l}\text { mit dem gekochten } \\
\text { Preßsaft versetzt }\end{array}$ \\
\hline b1 & 24 & 0,2 & 10 & 0,0136 & $\begin{array}{l}\text { mit dem frischen } \\
\text { Preßsaft versetzt }\end{array}$ \\
\hline b2 & 24 & 0,2 & 10 & 0,0034 & $\begin{array}{l}\text { mit dem gekochten } \\
\text { Preßsaft versetzt }\end{array}$ \\
\hline c1 & 51 & 0,2 & 10 & 0,0252 & $\begin{array}{l}\text { mit dem frischen } \\
\text { Preßsaft versetzt }\end{array}$ \\
\hline c2 & 51 & 0,2 & 10 & 0,0041 & $\begin{array}{l}\text { mit dem gekochten } \\
\text { Preßsaft versetzt }\end{array}$ \\
\hline
\end{tabular}

Versuch 2.

Dieser Versuch wurde zum Zwecke angestellt, um die Frage zu entscheiden, ob bei der Wirkung des Bambusschößlingpreßsaftes auf das hefenucleinsaure Natron die Bildung von Purinbasen stattfindet.

Probe a 1. $10 \mathrm{ccm}$ einer $2 \%$ igen Lösung von hefenucleinsaurem Natron $+10 \mathrm{ccm}$ Preßsaft $+1 \mathrm{ccm}$ Toluol. Nach 24 stündiger Digestion bei Bruttemperatur wurde die Mischung in ein Becherglas hineingegossen, nach Zusatz von 2 g Natriumacetat mit einer $20 \%$ igen Tanninlösung vollständig gefällt, abfiltriert und die Tanninfällung gründlich mit einer 10\% igen Natriumacetatlösung ausgewaschen. Man vereinigte das Filtrat mit dem Waschwasser, entfernte den Überschuß von Tannin durch Zusatz von Bleiessig, filtrierte $a b$ und befreite das Filtrat von überschüssigem Blei mittels Schwefelwasserstoffs. Die bleifreie Flüssigkeit wurde im Vakuum bei $35-38^{\circ}$ auf ein kleines Volumen eingeengt und mit einer ammoniakalischen Silberlösung ausgefällt.

Daß diese Silberfällung im wesentlichen aus den Silberverbindungen der Purinbasen bestand, braucht nicht hervor- 
gehoben zu werden. Die Silberfällung wurde nun mit Wasser ammoniakfrei gewaschen und der Stickstoffbestimmung nach Kjeldahl unterworfen. Es wurden gefunden: 6,4 mg N.

Probe a2. (Kontrollprobe.) $10 \mathrm{ccm}$ einer $2 \%$ igen Lösung von hefenucleinsaurem Natron $+10 \mathrm{ccm}$ gekochter Preßsaft $+1 \mathrm{ccm}$ Toluol. Nach 24 stündigem Stehenlassen im Brutraum wurde die Mischung auf die gleiche Weise auf Purinbasenstickstoff verarbeitet, wie bei Probe a1. Es wurden gefunden: $2,5 \mathrm{mg} \mathrm{N}$.

Probe b1. $10 \mathrm{ccm}$ einer 2\% igen Lösung von hefenucleinsaurem Natron $+10 \mathrm{ccm}$ Preßsaft $+1 \mathrm{ccm}$ Toluol. Die Mischung wurde 24 Stunden lang in den Brutschrank gestellt und dann auf Purinbasenstickstoff verarbeitet. Es wurden gefunden: $6,7 \mathrm{mg} \mathrm{N}$.

Probe b2. (Kontrollprobe.) $10 \mathrm{ccm}$ einer $2 \%$ igen Lösung von hefenucleinsaurem Natron $+10 \mathrm{ccm}$ gekochter Preßsaft $+1 \mathrm{ccm}$ Toluol. Die Mischung wurde nach 24 stündiger Digestion bei Bruttemperatur in gleicher Weise behandelt, wie bei den sonstigen Proben. Es wurden gefunden: $2,7 \mathrm{mg} \mathrm{N}$.

Bei allen Proben fielen die Strichkultur auf festem Nährboden und die Impfkultur in Bouillonlösung völlig negativ aus.

Die folgende Tabelle gibt einen Überblick über die geschilderten Versuchsergebnisse.

Tabelle 2.

\begin{tabular}{|c|c|c|c|c|c|}
\hline $\begin{array}{c}\text { Nummer } \\
\text { der } \\
\text { Proben }\end{array}$ & $\begin{array}{l}\text { Dauer } \\
\text { der Di- } \\
\text { gestion } \\
\text { in Std. }\end{array}$ & $\begin{array}{c}\text { Menge des } \\
\text { verwendeten } \\
\text { nucleinsauren } \\
\text { Natrons } \\
\text { in } \mathrm{g}\end{array}$ & \begin{tabular}{|c|} 
Menge \\
des ver- \\
wendeten \\
Preßsaftes \\
in ccm
\end{tabular} & $\begin{array}{l}\text { Menge des } \\
\text { gefundenen } \\
\text { Purinbasen- } \\
\text { stickstoffs } \\
\text { in mg }\end{array}$ & Bemerkungen \\
\hline a1 & 24 & 0,2 & 10 & 6,4 & $\begin{array}{l}\text { mit dem frischen } \\
\text { Preßsaft versetzt }\end{array}$ \\
\hline a2 & 24 & 0,2 & 10 & 2,5 & $\begin{array}{l}\text { mit dem gekochten } \\
\text { Preßsaft versetzt }\end{array}$ \\
\hline b1 & 24 & 0,2 & 10 & 6,7 & $\begin{array}{l}\text { mit dem frischen } \\
\text { Preßsaft versetzt }\end{array}$ \\
\hline b2 & 24 & 0,2 & 10 & 2,7 & $\begin{array}{l}\text { mit dem gekochten } \\
\text { Preßsaft versetzt }\end{array}$ \\
\hline
\end{tabular}


Aus der Tabelle geht hervor, daß bei der Digestion des hefenucleinsauren Natrons mit frischem Bambusschößlingpreßsafte der Purinbasenstickstoff eine bedeutende Zunahme erfährt. Der in den Kontrollproben aufgefundene Stickstoff gehört zweifellos Purinbasen an, welche bereits in Bambusschößlinge vorgebildet sind; ich erinnere hier an die Beobachtungen von Y. Kosai ${ }^{1}$ ) und von G. Totani. ${ }^{2}$ )

Aus den angeführten Versuchsreihen ergibt sich die Tatsache, daß der Preßsaft aus Bambusschößlingen die Spaltung der zugesetzten Nucleinsäure in Purinbasen und Phosphorsäure bewirkt. Daß diese Spaltung fermentativer Natur ist, ist dadurch erwiesen, daß der vorher wirksame Preßsaft nach dem Erhitzen zum Sieden sich völlig indifferent gegen Nucleinsäure verhält.

\section{Desamidasen.}

In neuerer Zeit sind Tatsachen bekannt geworden, welche dafür sprechen, daß Desamidasen sich großer Verbreitung im Pflanzenreich erfreuen.

K. Shibata $\left.{ }^{3}\right)$ konnte zeigen, daß im Mycelium von Aspergillus niger ein Ferment vorhanden ist, welches Ammoniak aus Harnstoff, Biuret, Acetamid und Asparagin abzuspalten vermag. Durch schöne Untersuchungen wurde von Felix Ehrlich ${ }^{4}$ ) festgestellt, dảß bei der Gärung mit Hefe das Leucin und Isoleucin eine Spaltung in die entsprechenden Amylalkohole sowie in Ammoniak und Kohlensäure erleiden. Im Anschluß an diese Untersuchungen stellte J. Effront ${ }^{5}$ ) eine Anzahl von Versuchen mit der Bierhefe an und fand, daß in obergärigen und Äerohefen, sowie in Amylobakter butylicus ein Ferment, "Amidase», vorkommt, welches die Fähigkeit

1) Y. Kosai, l. c.

2) G. Totani, l. c.

3) K. Shibata, Hofmeisters Beiträge, Bd. 5, S. 384.

4) F. Ehrlich, Berichte d. deutsch. chem. Ges., Bd. 40, S. 1027 und S. 2542 .

5) J. Effront, Compt. rend. d. l'acad. d. scienc., Bd. 146, S. 779. 
besitzt, Asparagin, Asparaginsäure, Glutaminsäure und Leucin in Ammoniak und flüchtige Fettsäuren überzuführen. Daß aber diese Amidase merkwürdigerweise nicht immer in untergärigen Hefen aufzufinden ist, scheint er durch besondere Versuche dargetan zu haben, denn er schließt seine Abhandlung mit dem Wort: "Dans les levures de fermentation basse, la présence de cet enzyme nous paraît incertaine."

T. Takeuchi, ${ }^{1}$ ) der auch bei den höheren Pflanzen dieser Frage seine Aufmerksamkeit zuwandte, gibt an, daß es ihm gelang, ein Ferment in Sojabohnen, Glytine hispida, nachzuweisen, welches die Abspaltung des Ammoniaks aus Harnstoff und Asparagin bewirkt.

Bei den nachstehenden Versuchen fand auch der Preßsaft, welcher nach dem oben angegebenen Verfahren aus Bambusschößlingen hergestellt wurde, Anwendung. Die Bestimmung des Ammoniaks ${ }^{2}$ ) geschah stets nach der Methode von Krüger und Reich.

\section{Versuch 1.}

Probe a1. 0,2 $\mathrm{g}$ Harnstoff wurden in $10 \mathrm{ccm}$ Wasser gelöst, mit $10 \mathrm{ccm}$ Preßsaft versetzt und die Mischung unter Zusatz von $1 \mathrm{ccm}$ Toluol für 20 Stunden in den Brutschrank gestellt. Es hatten sich gebildet: $5,1 \mathrm{mg} \mathrm{NH}_{3}$.

Probe a 2 (Kontrollprobe). 0,2 g Harnstoff wurden in $10 \mathrm{ccm}$ Wasser gelöst und $10 \mathrm{ccm}$ gekochter Preßsaft nebst $1 \mathrm{ccm}$ Toluol zugesetzt. Nach 20 stündiger Digestion bei Bruttemperatur war keine Spur von $\mathrm{NH}_{3}$ in diesem Gemisch nachzuweisen.

Probe b1. 0,2 $\mathrm{g}$ Harnstoff in $10 \mathrm{ccm}$ Wasser gelöst + $10 \mathrm{ccm}$ Preßsaft $+1 \mathrm{ccm}$ Toluol. 36 stündige Digestion bei Bruttemperatur. Es hatten sich gebildet: $12,3 \mathrm{mg} \mathrm{NH}$.

Probe b2 (Kontrollprobe). 0,2 g Harnstoff in $10 \mathrm{ccm}$ Wasser gelöst $+10 \mathrm{ccm}$ gekochter Prelssaft $+1 \mathrm{ccm}$ Toluol. 36 stündige Digestion im Brutraum. Keine Spur von Ammoniak im Gemisch.

1) T. Takeuchi. Journ. of the coll. of agric., Tokyo, Bd. 1, S. 1-14.

$\left.{ }^{2}\right)$ War Eiweiß in der Probe vorhanden, so wurde das Ammoniak immer nach dem Enteiweißen bestimmt. 
Probe c1. 0,2 g Harnstoff in $10 \mathrm{ccm}$ Wasser gelöst + $10 \mathrm{ccm}$ Preßsaft $+1 \mathrm{ccm}$ Toluol. 51 stündige Digestion im Brutraum. Es hatten sich gebildet: $15,8 \mathrm{mg} \mathrm{NH}$.

Probe c2 (Kontrollprobe). 0,2 $\mathrm{g}$ Harnstoff in $10 \mathrm{ccm}$ Wasser gelöst $+10 \mathrm{ccm}$ gekochter Preßsaft $+1 \mathrm{ccm}$ Toluol. 51 stündige Digestion im Brutraum. Im Gemisch war keine Spur von $\mathrm{NH}_{3}$ nachzuweisen.

Alle Proben erwiesen sich als steril.

Die erhaltenen Befunde sind der Übersicht wegen in der folgenden Tabelle zusammengestellt.

Tabelle 3.

\begin{tabular}{|c|c|c|c|c|c|}
\hline $\begin{array}{c}\text { Nummer } \\
\text { der } \\
\text { Proben }\end{array}$ & $\begin{array}{c}\text { Dauer } \\
\text { der } \\
\text { Digestion } \\
\text { in Std. }\end{array}$ & $\begin{array}{c}\text { Menge } \\
\text { des ver- } \\
\text { wendeten } \\
\text { Harnstoffs } \\
\text { in } \mathbf{g}\end{array}$ & $\begin{array}{c}\text { Menge } \\
\text { des ver- } \\
\text { wendeten } \\
\text { Preßsaftes } \\
\text { in ccm }\end{array}$ & $\begin{array}{c}\text { Menge } \\
\text { des } \\
\text { gebildeten } \\
\mathrm{NH}_{\mathbf{s}} \\
\text { in } \mathrm{mg}\end{array}$ & Bemerkungen \\
\hline a1 & 20 & 0,2 & 10 & 5,1 & $\begin{array}{l}\text { mit dem frischen } \\
\text { Preßsaft versetzt }\end{array}$ \\
\hline 22 & 20 & 0,2 & 10 & 0 & $\begin{array}{l}\text { mit dem gekochten } \\
\text { Preßsaft versetzt }\end{array}$ \\
\hline b1 & 36 & 0,2 & 10 & 12,3 & $\begin{array}{l}\text { mit dem frischen } \\
\text { Preßsaft versetzt }\end{array}$ \\
\hline b2 & 36 & 0,2 & 10 & 0 & $\begin{array}{l}\text { mit dem gekochten } \\
\text { Preßsaft versetzt }\end{array}$ \\
\hline c1 & 51 & 0,2 & 10 & 15,8 & $\begin{array}{l}\text { mit dem frischen } \\
\text { Preßsaft versetzt }\end{array}$ \\
\hline c2 & 51 & 0,2 & 10 & 0 & $\begin{array}{l}\text { mit dem gekochten } \\
\text { Preßsaft versetzt }\end{array}$ \\
\hline
\end{tabular}

Bei allen Proben, a1, b1 und c1, ist somit mit Sicherheit erwiesen, daß der Bambusschößlingpreßsaft über ein ureaseähnliches Ferment verfügt, welches bei neutraler Reaktion rasch den Harnstoff angreift und zur Bildung von Ammoniak führt.

\section{Versuch 2.}

Probe a1. 0,2 g Asparagin in $10 \mathrm{ccm}$ Wasser gelöst + $10 \mathrm{ccm}$ Preßsaft $+1 \mathrm{ccm}$ Toluol. 20 stündige Digestion bei Bruttemperatur. Die Menge des gebildeten Ammoniaks $=0,5 \mathrm{mg}$.

Probe a 2 (Kontrollprobe). 0,2 g Asparagin in $10 \mathrm{ccm}$ Wasser gelöst $+10 \mathrm{ccm}$ gekochter Preßsaft $+1 \mathrm{ccm}$ Toluol. 
20 stündige Digestion im Brutraum. Die Mischung war völlig frei von Ammoniak.

Probe b1. 0,2 g Asparagin in $10 \mathrm{ccm}$ Wasser gelöst + $10 \mathrm{ccm}$ Preßsaft $+1 \mathrm{ccm}$ Toluol. 36 stündiges Stehenlassen im Brutraum. Die Menge des gebildeten Ammoniaks $=0,5 \mathrm{mg}$.

Probe b2 (Kontrollprobe). 0,2 $\mathrm{g}$ Asparagin in $10 \mathrm{ccm}$ Wasser gelöst $+10 \mathrm{ccm}$ gekochter Preßsaft $+1 \mathrm{ccm}$ Toluol. Das Gemisch war nach 36 stündiger Aufbewahrung bei Bruttemperatur völlig frei von Ammoniak.

Probe c1. 0,2 g Asparagin in $10 \mathrm{ccm}$ Wasser gelöst + $10 \mathrm{ccm}$ Preßsaft $+1 \mathrm{ccm}$ Toluol. 51 stündige Digestion bei Bruttemperatur. Die Menge des gebildeten Ammoniaks $=0,3 \mathrm{mg}$.

Probe c2 (Kontrollprobe). 0,2 g Asparagin in $10 \mathrm{ccm}$ Wasser gelöst $+10 \mathrm{ccm}$ gekochter Preßsaft $+1 \mathrm{ccm}$ Toluol. 51 stündige Digestion im Brutraum. Das Gemisch war ganz. frei von Ammoniak.

Bei allen Proben wurde jede Einwirkung von Mikroorganismen vollkommen ausgeschlossen.

Die gewonnenen Resultate sind übersichtlich in die folgende Tabelle eingetragen.

Tabelle 4

\begin{tabular}{|c|c|c|c|c|c|}
\hline $\begin{array}{c}\text { Nummer } \\
\text { der } \\
\text { Proben }\end{array}$ & \begin{tabular}{|c|} 
Dauer \\
der \\
Digestion \\
in Std.
\end{tabular} & $\begin{array}{c}\text { Menge } \\
\text { des ver- } \\
\text { wendeten } \\
\text { Asparagins } \\
\text { in g }\end{array}$ & $\begin{array}{c}\text { Menge } \\
\text { des ver- } \\
\text { wendeten } \\
\text { Preßsaftes } \\
\text { in ccm }\end{array}$ & $\begin{array}{c}\text { Menge } \\
\text { des } \\
\text { gebildeten } \\
\text { Ammoniaks } \\
\text { in } \mathrm{mg}\end{array}$ & Bemerkungen \\
\hline a1 & 20 & 0,2 & 10 & $0, \overline{\mathbf{a}}$ & $\begin{array}{l}\text { mit dem frischen } \\
\text { Preßsaft versetzt }\end{array}$ \\
\hline a2 & 20 & 0,2 & 10 & 0 & $\begin{array}{l}\text { mit dem gekochten } \\
\text { Preßsaft versetzt }\end{array}$ \\
\hline b1 & 36 & 0,2 & 10 & 0,5 & $\begin{array}{l}\text { mit dem frischen } \\
\text { Preßsaft versetzt }\end{array}$ \\
\hline $\mathrm{b} 2$ & 36 & 0,2 & 10 & 0 & $\begin{array}{l}\text { mit dem gekochten } \\
\text { Preßsaft versetzt }\end{array}$ \\
\hline $\mathrm{c1}$ & 51 & 0,2 & 10 & 0,3 & $\begin{array}{l}\text { mit dem frischen } \\
\text { Preßsaft versetzt }\end{array}$ \\
\hline c2 & 51 & 0,2 & 10 & 0 & $\begin{array}{l}\text { mit dem gekochten } \\
\text { Preßsaft versetzt }\end{array}$ \\
\hline
\end{tabular}


Aus den Versuchsresultaten läßt sich erkennen, daß bei Einwirkung von Bambusschößlingpreßsaft das Asparagin eine sehr geringe Menge Ammoniak abgibt. Diese ammoniakabspaltende Wirkung aber geht dann dem Preßsaft ab, wenn er zum Sieden erhitzt wird.

Weiter nahm ich auch die Untersuchung über die Einwirkung von Bambusschößlingpreßsaft auf das Glykokoll vor, vermochte jedoch die Bildung von Ammoniak in keinem Fall zu beobachten.

Faßt man die Ergebnisse der vorliegenden Versuche zusammen, so ergibt sich:

1. In Bambusschößlingen findet sich ein Ferment, welches die Abspaltung von Ammoniak aus Harnstoff und Asparagin bewirkt.

2. Das Ferment greift das Glykokoll nicht an.

\section{Proteolytische Fermente.}

Bezüglich der proteolytischen Fermente in Bambusschößlingen konnte ich folgendes konstatieren: der auf die übliche Weise bereitete Preßsaft greift bei neutraler Reaktion und Bruttemperatur das Fibrin an. Diese Wirkung aber wird durch Kochhitze vollständig aufgehoben.

Die Untersuchung über die Natur des fibrinlösenden Fermentes in Bambusschößlingen und die Abbauprodukte, welche unter Einwirkung dieses Fermentes aus den verschiedenen Eiweißstoffen entstehen, ist bereits im Gang und ich behalte mir die Veröffentlichung der gewonnenen Resultate auf eine spätere ausführliche Mitteilung vor.

\section{Diastatische Fermente.}

Wie eine ganze Reihe von Forschern gezeigt haben, ist die Rolle, welche die diastatischen Fermente bei den Stoffwechselprozessen der Pflanzen - besonders der keimenden Samen - spielen, so bedeutend, daß ohne Vermittelung derselben die Verwertung der Polysaccharide überhaupt nicht möglich ist. Man begegnet den diastatischen Fermenten überall $\mathrm{da}$, wo das Bedürfnis vorhanden ist, die als Reservestoff ab-

Hoppe-Seyler's Zeitschrift f. physiol. Chemie. LXXV. 
gelagerte Stärke in lösliche Substanz umzuwandeln und durch alle Teile des Pflanzenkörpers transportabel zu machen.

Ich habe durch anderweitige Versuche festgestellt, daß im aus jungen Bambusschößlingen erhaltenen Preßsaft ein stärkeähnlicher Körper enthalten ist, welcher durch Jod schön blau gefärbt wird; ich habe ferner beobachtet, daß beim Stehenlassen des Preßsaftes die Neubildung von Zucker auf Kosten des betreffenden Körpers sich vollzieht; ich konnte endlich zeigen, daß diese Zuckerbildung in hohem Grade beeinträchtigt wird, wenn der Preßsaft gekocht und dann sorgfältig von der Einwirkung der Mikroorganismen ausgeschlossen wird. Diese Tatsachen gestatten schon die Annahme, daß ein diastatisches Ferment oder eine Gruppe von derartigen Fermenten sich in Bambusschößlingen finden, welche die Hydrolyse der Polysaccharide herbeiführen. Wie aus folgenden Versuchen ersichtlich ist, hat sich diese Annahme über Erwarten bestätigt.

\section{Versuch 1}

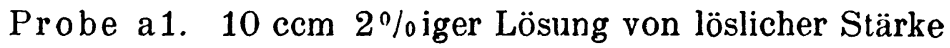
$+10 \mathrm{ccm}$ Bambusschößlingpreßsaft $+1 \mathrm{ccm}$ Toluol. 41 stündige Digestion im Brutraum. Das Gemisch verliert die Fähigkeit, durch Jod gefärbt $\mathrm{zu}$ werden.

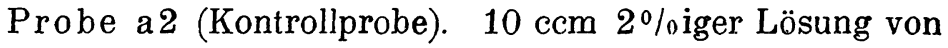
löslicher Stärke $+10 \mathrm{ccm}$ gekochter Preßsaft $+1 \mathrm{ccm}$ Toluol. 41 stündige Aufbewahrung im Brutraum. Das Gemisch färbt sich blau auf Zusatz von einer Jod-Jodkalilösung.


$+10 \mathrm{ccm}$ Preßsaft $+1 \mathrm{ccm}$ Toluol. 15stündiges Verweilen im Brutraum. Keine Jodreaktion.

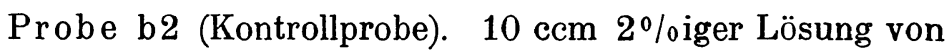
löslicher Stärke $+10 \mathrm{ccm}$ gekochter Preßsaft $+1 \mathrm{ccm}$ Toluol. 15stündiges Stehenbleiben bei Bruttemperatur. Starke Jodreaktion.

Alle Proben waren vollkommen frei von Mikroorganismen.

Die Ergebnisse sind übersichtlich in folgender Tabelle zusammengestellt. 
Tabelle 5 .

\begin{tabular}{|c|c|c|c|c|c|}
\hline $\begin{array}{l}\text { Nummer } \\
\text { der } \\
\text { Proben }\end{array}$ & $\begin{array}{c}\text { Dauer } \\
\text { der } \\
\text { Digestion } \\
\text { in Std. }\end{array}$ & $\begin{array}{c}\text { Menge der } \\
2 \% \text { igen } \\
\text { Stärke- } \\
\text { lösung } \\
\text { in ccm }\end{array}$ & $\begin{array}{c}\text { Menge } \\
\text { des ver- } \\
\text { wendeten } \\
\text { Preßsaftes } \\
\text { in ccm }\end{array}$ & $\begin{array}{l}\text { Jod- } \\
\text { reaktion }\end{array}$ & Bemerkungen \\
\hline a1 & 41 & 10 & 10 & - & $\begin{array}{l}\text { mit dem frischen } \\
\text { Preßsaft versetzt }\end{array}$ \\
\hline 22 & 41 & 10 & 10 & + & $\begin{array}{l}\text { mit dem gekochten } \\
\text { Preßsaft versetzt }\end{array}$ \\
\hline b1 & 15 & 10 & 10 & - & $\begin{array}{l}\text { mit dem frischen } \\
\text { Preßsaft versetzt }\end{array}$ \\
\hline b2 & 15 & 10 & 10 & + & $\begin{array}{l}\text { mit dem gekochten } \\
\text { Preßsaft versetzt }\end{array}$ \\
\hline
\end{tabular}

Versuch 2.

Diese Versuche wurden zu dem Zwecke angestellt, um die Bildung von Zucker bei der Digestion der Stärkelösung mit dem Bambusschößlingpreßsaft zu verfolgen. Zur Zuckerbestimmung bediente îch mich der Methode von Gabriel Bertrand $\left.{ }^{1}\right)$ und berechnete den gefundenen Zucker als Traubenzucker.

Probe a1. $10 \mathrm{ccm} 2 \%$ iger Lösung von löslicher Stärke $+10 \mathrm{ccm}$ des aus Bambusschößlingen hergestellten Preßsaftes $+1 \mathrm{ccm}$ Toluol. 15stündige Digestion im Brutraum. Im Gemisch wurden gefunden: 66,0 mg Zucker.

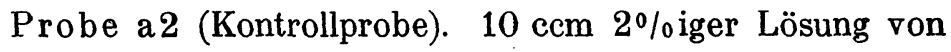
löslicher Stärke $+10 \mathrm{ccm}$ gekochter Preßsaft $+1 \mathrm{ccm}$ Toluol. 15stündige Digestion im Brutraum. Im Gemisch wurden gefunden: $20,9 \mathrm{mg}$ Zucker.

Probe a 3. $10 \mathrm{ccm}$ Preßsaft $+1 \mathrm{ccm}$ Toluol. 15stündige Digestion im Brutraum. Es wurden gefunden: $11,5 \mathrm{mg}$ Zucker.

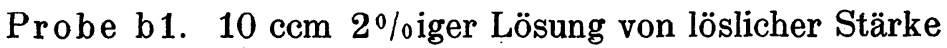
$+10 \mathrm{ccm}$ Preßsaft $+1 \mathrm{ccm}$ Toluol. 36stündiges Stehenlassen im Brutraum. Es wurden gefunden: 66,0 mg Zucker.

1) G. Bertrand, Guide pour les manipulations de chimie biologique. Paris 1910, S. 67. 


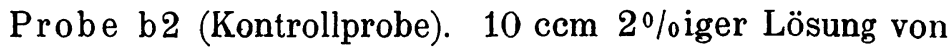
löslicher Stärke $+10 \mathrm{ccm}$ gekochter Preßsaft $+1 \mathrm{ccm}$ Toluol. 36 stündiges Stehen im Brutraum. Es wurden gefunden : 28,2 mg Zucker.

Probe b3. $10 \mathrm{ccm}$ Preßsaft $+1 \mathrm{ccm}$ Toluol. 36stündiges Stehen im Brutraum. Es wurden gefunden: 11,5 mg Zucker. Alle Proben erwiesen sich als steril.

Tabelle 6.

\begin{tabular}{|c|c|c|c|c|c|}
\hline $\begin{array}{c}\text { Nummer } \\
\text { der } \\
\text { Proben }\end{array}$ & $\begin{array}{c}\text { Dauer } \\
\text { der } \\
\text { Digestion } \\
\text { in Std. }\end{array}$ & $\begin{array}{l}\text { Menge der } \\
2 \% \text { igen } \\
\text { Stärke- } \\
\text { lösung } \\
\text { in ccm }\end{array}$ & $\begin{array}{c}\text { Menge } \\
\text { des ver- } \\
\text { wendeten } \\
\text { Preßsaftes } \\
\text { in ccm }\end{array}$ & $\begin{array}{c}\text { Menge } \\
\text { des ge- } \\
\text { fundenen } \\
\text { Zuckers } \\
\text { in mg }\end{array}$ & Bemerkungen \\
\hline a1 & 15 & 10 & 10 & 66,0 & $\begin{array}{l}\text { mit dem frischen } \\
\text { Preßsaft versetzt }\end{array}$ \\
\hline a2 & 15 & 10 & 10 & 20,9 & $\begin{array}{c}\text { mit dem gekochten } \\
\text { Preßsaft versetzt }\end{array}$ \\
\hline a3 & 15 & 0 & 10 & 11,5 & $\begin{array}{l}\text { ohne Zusatz von } \\
\text { Stärkelösung }\end{array}$ \\
\hline b1 & 36 & 10 & 10 & 66,0 & $\begin{array}{l}\text { mit dem frischen } \\
\text { Preßsaft versetzt }\end{array}$ \\
\hline b2 & 36 & 10 & 10 & 28,2 & $\begin{array}{l}\text { mit dem gekochten } \\
\text { Preßsaft versetzt }\end{array}$ \\
\hline b3 & 36 & 0 & 10 & 11,5 & $\begin{array}{l}\text { ohne Zusatz von } \\
\text { Stärkelösung }\end{array}$ \\
\hline
\end{tabular}

Die Ergebnisse stellen es außer Zweifel, daß die Digestion der Stärkelösung mit dem Bambusschößlingpreßsaft die Bildung von Zucker zur Folge hat. Daß bei den Kontrollproben, a 2 und b2, die Zuckerbildung nicht gänzlich verhindert war, kann vielleicht dadurch erklärt werden, daß die Kochdauer zu kurz war, um ein zuckererzeugendes Agens vollständig zu vernichten. Der Zucker, welcher bei den Proben a3 und b3 gefunden wurde, war teils sicher im Preßsaft präformiert, teils läßt er sich aus der darin vorhandenen Stärke oder den ihr nahestehenden Körpern ableiten.

An dieser Stelle sei hervorgehoben, daß es Herrn Dr. Junji Yoshikawa ${ }^{1}$ ) gelang, einen Zucker aus den Bambus-

1) J. Yoshikawa, Die Arbeit ist noch nicht veröffentlicht. 
schößlingen $\mathrm{zu}$ isolieren, welcher folgende Eigenschaften zeigt: er reduziert Kupferoxyd in alkalischer Lösung, dreht das polarisierte Licht nach links, vergärt mit Hefe, gibt schöne Seliwan offsche Reaktion (nach der Vorschrift von Ofner ausgeführt) und liefert beim Erwärmen mit essigsaurem Phenylhydrazin ein Osazon, welches die von Glukosazon geforderte Krystallform und den Schmelzpunkt von $204-205^{\circ} \mathrm{C}$. hat. Die Untersuchungen inbetreff der folgenden wichtigsten Fragen: ob der erwähnte linksdrehende Zucker mit Fruktose identisch ist, ob außer dem linksdrehenden Zucker noch andere Zuckerarten in Bambusschößlingen nachzuweisen sind, aus welcher Muttersubstanz und unter welcher Bedingung der linksdrehende Zucker entsteht? sind schon von Herrn J. Yoshikawa in Angriff genommen, aber noch nicht zu Ende geführt. Ausführliche Mitteilung über dieselben bleibt deshalb ihm vorbehalten.

Daß das Endprodukt, welches bei Einwirkung vom Bambusschößlingpreßsaft aus zugesetzter Stärke entsteht, d-Glukose ist, habe ich mit aller Sicherheit erwiesen.

Das Gesamtergebnis der geschilderten Versuche führt zu der Folgerung, daB in Bambusschößlingen Fermente vorkommen, welche bei neutraler Reaktion Stärke abbauen. Ob diese Fermente aus einem Gemisch von Diastase, Maltase und Glukase bestehen, ob die Mengenverhältnisse dieserFermente je nach demWachstumsstadium der BambusschöBlinge eine Veränderung erfahren, darüber läßt sich vorderhand nichts Sicheres sagen.

\section{Ein Amygdalin spaltendes Ferment.}

Außer den oben beschriebenen fermentativen Wirkungen besitzt der Bambusschößlingpreßsaft die Fähigkeit, den Zucker aus Amygdalin abzuspalten. Diese Spaltung zu verfolgen und die Spaltprodukte möglichst genau zu charakterisieren, das bildet den Gegenstand der folgenden Mitteilung.

Versuch 1.

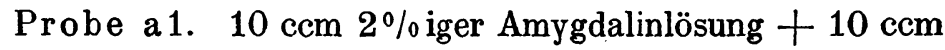
Bambusschößlingpreßsaft $+1 \mathrm{ccm}$ Toluol. 40 stündiges Stehen 
im Brutraum. Der Geruch nach Benzaldehyd war sehr stark. Das Gemisch wurde nun der Destillation unterworfen; das so erhaltene Destillat gab schöne Berlinerblau- und GuajaktinkturKupfersulfatreaktion. Somit wurde Blausäure mit Sicherheit im Gemisch nachgewiesen.


lösung $+10 \mathrm{ccm}$ gekochter Preßsaft $+1 \mathrm{ccm}$ Toluol. 40 stündiges Stehen im Brutraum. Kein Geruch nach Benzaldehyd. Das Gemisch wird destilliert und das Destillat auf Blausäure geprüft : die Guajaktinktur-Kupfersulfatprobe trat sehr schwach ein, die Berlinerblaureaktion dagegen fiel völlig negativ aus. Es war mithin Blausäure im Gemisch nicht vorhanden.

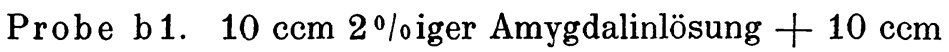
Preßsaft $+1 \mathrm{ccm}$ Toluol. 52 stündige Digestion im Brutraum. Starker Geruch nach Benzaldehyd. Das aus dem Gemisch gewonnene Destillat gab intensive Guajaktinktur-Kupfersulfatund Berlinerblaureaktion. Es wurde also das Vorkommen der Blausäure im Gemisch festgestellt.

Probe b2 (Kontrollprobe). $10 \mathrm{ccm} \mathrm{2 \%}$ iger Amygdalinlösung $+10 \mathrm{ccm}$ gekochter Preßsaft $+1 \mathrm{ccm}$ Toluol. 52 stündige Digestion im Brutraum. Der Geruch nach Benzaldehyd war nicht, wahrnehmbar. Das aus dem Gemisch hergestellte Destillat gab sehr schwache Guajaktinktur-Kupfersulfatreaktion; die Berlinerblaureaktion aber war ganz negativ. Es hatte sich also keine Blausäure im Gemisch gebildet.

Alle Proben waren ganz steril (s. Tab. 7).

Versuch 2.

Zur Feststellung der Zuckerbildung aus Amygdalin bei Einwirkung von Bambusschößlingpreßsaft wurde dieser Versuch angestellt. Die Zuckerbestimmung erfolgte hier auch genau nach der Vorschrift von G. Bertrand.

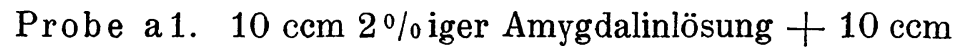
Bambusschößlingpreßsaft $+1 \mathrm{ccm}$ Toluol. 48 stündige Digestion im Brutraum. Es wurden gefunden: $87,3 \mathrm{mg}$ Zucker.

Probe a 2 (Kontrollprobe). $10 \mathrm{ccm} \mathrm{2 \%}$ iger Amygdalinlösung $+10 \mathrm{ccm}$ gekochter Preßsaft $+1 \mathrm{ccm}$ Toluol. 48 stündige Digestion im Brutraum. Es wurden gefunden: 42,1 mg Zucker. 
Tabelle 7.

\begin{tabular}{c|c|c|c|c|c|c}
\hline $\begin{array}{c}\text { Nummer } \\
\text { der }\end{array}$ & $\begin{array}{c}\text { Dauer } \\
\text { der } \\
\text { Di- } \\
\text { gestion } \\
\text { in Std. }\end{array}$ & $\begin{array}{c}\text { Menge } \\
\text { der } \\
2 \% \text { igen } \\
\text { Amygdalin- } \\
\text { lösung } \\
\text { in ccm }\end{array}$ & $\begin{array}{c}\text { Menge } \\
\text { des } \\
\text { verwen- } \\
\text { deten } \\
\text { Preß- } \\
\text { saftes } \\
\text { in ccm }\end{array}$ & $\begin{array}{c}\text { Ber- } \\
\text { liner- } \\
\text { blau- } \\
\text { reak- } \\
\text { tion }\end{array}$ & $\begin{array}{c}\text { Guajak- } \\
\text { tinktur- } \\
\text { Kupfer- } \\
\text { sulfat- } \\
\text { reaktion }\end{array}$ & Bemerkungen \\
a1 & 40 & 10 & 10 & + & + & $\begin{array}{c}\text { mit dem frischen } \\
\text { Preßsaft versetzt } \\
\text { a2 }\end{array}$ \\
b1 & 52 & 10 & 10 & - & $\begin{array}{c}\text { sehr } \\
\text { mit dem gekochten }\end{array}$ \\
b2 & 52 & 10 & 10 & - & $\begin{array}{c}\text { Preßsaft versetzt } \\
+ \\
\text { mit dem frischen } \\
\text { Preßsaft versetzt } \\
\text { schwach } \\
\text { mit dem gekochten } \\
\text { Preßsaft versetzt }\end{array}$
\end{tabular}

Probe a 3. $10 \mathrm{ccm}$ Preßsaft $+1 \mathrm{ccm}$ Toluol. 48 stündige Digestion im Brutraum. Es wurden gefunden: 57,1 mg Zucker. Alle Proben waren ganz steril.

Tabelle 8.

\begin{tabular}{c|c|c|c|c|c}
\hline $\begin{array}{c}\text { Nummer } \\
\text { der } \\
\text { Proben }\end{array}$ & $\begin{array}{c}\text { Dauer } \\
\text { der } \\
\text { Digestion } \\
\text { in Std. }\end{array}$ & $\begin{array}{c}\text { Menge } \\
\text { der 2\% } \% \text { igen } \\
\text { Amygdalin- } \\
\text { lösung } \\
\text { in ccm }\end{array}$ & $\begin{array}{c}\text { Menge } \\
\text { des ver- } \\
\text { wendeten } \\
\text { Preßsaftes } \\
\text { in ccm }\end{array}$ & $\begin{array}{c}\text { Menge } \\
\text { des ge- } \\
\text { fundenen } \\
\text { Zuckers } \\
\text { in mg }\end{array}$ & Bemerkungen \\
\hline a1 & 48 & 10 & 10 & 87,3 & $\begin{array}{c}\text { mit dem frischen } \\
\text { Preßsaft versetzt } \\
\text { mit dem gekochten } \\
\text { Preßsaft versetzt } \\
\text { ahne Zusatz von } \\
\text { Amygdalin }\end{array}$
\end{tabular}

Aus den obigen Versuchen geht deutlich hervor, daß die Bambusschößlinge ein Ferment besitzen, welches Amygdalin unter Bildung von Benzaldehyd, Blausäure und Traubenzucker spaltet.

\section{Ein Salicin spaltendes Ferment.}

Versuch 1.

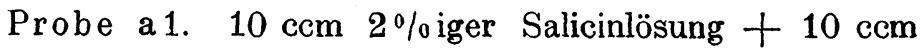
Bambusschößlingpreßsaft $+1 \mathrm{ccm}$ Toluol. 40 stündiges Ver- 
bleiben im Brutraum. Das Gemisch wurde nun mit Äther extrahiert, der Ätherauszug in einem Becherglas freiwilligem Verdunsten überlassen und der Rückstand mit Wasser aufgenommen. Die wässerige Lösung färbte sich sofort blau auf Zusatz von einem Tropfen Eisenchloridlösung. Es wurde somit die Abspaltung des Saligenins aus Salicin nachgewiesen.

Probe 2 a (Kontrollprobe). $10 \mathrm{~cm} \mathrm{2 \%}$ \% iger Salicinlösung $+10 \mathrm{ccm}$ gekochter Preßsaft $+1 \mathrm{ccm}$ Toluol. 40 stündiges Verbleiben in Brutraum. Das Gemisch wurde auf die gleiche Weise behandelt wie bei Probe a 1. Das negative Ausfallen der Eisenchloridreaktion beweist, daß das Salicin hier keine Veränderung erfuhr.

Bei allen Proben war die Mitwirkung der Mikroorganismen ausgeschlossen.

Tabelle 9 .

\begin{tabular}{c|c|c|c|c|c}
\hline $\begin{array}{c}\text { Nummer } \\
\text { der }\end{array}$ & $\begin{array}{c}\text { Dauer } \\
\text { der } \\
\text { Digestion } \\
\text { in Std. }\end{array}$ & $\begin{array}{c}\text { Menge der } \\
\text { 2\%igen } \\
\text { Salicin- } \\
\text { lösung } \\
\text { in ccm }\end{array}$ & $\begin{array}{c}\text { Menge } \\
\text { des ver- } \\
\text { wendeten } \\
\text { Preßsaftes } \\
\text { in ccm }\end{array}$ & $\begin{array}{c}\text { Eisen- } \\
\text { chlorid- } \\
\text { reaktion }\end{array}$ & Bemerkungen \\
\hline a1 & 40 & 10 & 10 & + & $\begin{array}{c}\text { mit dem frischen } \\
\text { Preßsaft versetzt } \\
\text { mit dem gekochten } \\
\text { Preßsaft versetzt }\end{array}$
\end{tabular}

Versuch 2.

Bei diesem Versuche wurde der Einfluß des Bambusschößlingpreßsaftes auf die Bildung von Zucker aus Salicin untersucht und der Zucker nach dem Verfahren von Gabriel Bertrand bestimmt.

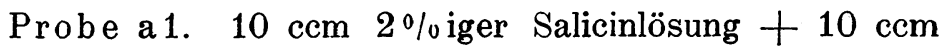
Bambusschößlingpreßsaft $+1 \mathrm{ccm}$ Toluol. 48 stündiges Stehen im Brutraum. Es wurden gefunden: $86,0 \mathrm{mg}$ Zucker.

Probe a 2 (Kontrollprobe). $10 \mathrm{ccm} 2 \%$ iger Salicinlösung $+10 \mathrm{ccm}$ gekochter Preßsaft $+1 \mathrm{ccm}$ Toluol. 48 stündiges Stehen im Brutraum. Es wurden gefunden: 44,8 mg Zucker.

Probe a 3. $10 \mathrm{ccm}$ Preßsaft $+1 \mathrm{ccm}$ Toluol. 48 stündiges Stehen im Brutraum. Es wurden gefunden: 64,6 mg Zucker. 
Bei den Proben war die Einwirkung von Mikroorganismen ausgeschlossen.

Tabelle 10.

\begin{tabular}{c|c|c|c|c|c}
\hline $\begin{array}{c}\text { Nummer } \\
\text { der } \\
\text { Proben }\end{array}$ & $\begin{array}{c}\text { Dauer } \\
\text { der } \\
\text { Digestion } \\
\text { in Std. }\end{array}$ & $\begin{array}{c}\text { Menge der } \\
2^{\circ} \text { o igen } \\
\text { Salicin- } \\
\text { lösung } \\
\text { in ccm }\end{array}$ & $\begin{array}{c}\text { Menge } \\
\text { des ver- } \\
\text { wendeten } \\
\text { Preßsaftes } \\
\text { in ccm }\end{array}$ & $\begin{array}{c}\text { Menge } \\
\text { des ge- } \\
\text { fundenen } \\
\text { Zuckers } \\
\text { in mg }\end{array}$ & Bemerkungen \\
\hline a1 & 48 & 10 & 10 & 86,0 & $\begin{array}{c}\text { mit dem frischen } \\
\text { Preßsaft versetzt } \\
\text { mit dem gekochten } \\
\text { Preßsaft versetzt } \\
\text { ohne Znsatz von } \\
\text { Preßsaft }\end{array}$ \\
\hline
\end{tabular}

Aus der Tabelle ergibt sich, daß bei Probe a 1 der Zucker in viel größerer Menge auftrat, als bei den übrigen. Diese Tatsache wird genügen, zu zeigen, daß dem Preßsaft aus Bambusschößlingen die Fähigkeit zukommt, den Zucker aus Salicin abzuspalten. Daß diese Fähigkeit an das Vorkommen von einem Ferment gebunden ist, kann gewiß nicht bezweifelt werden, denn sie wird durch die Hitze stark beeinträchtigt.

Die erwähnten Versuche lehren also, daß in Bambusschößlingen ein Ferment enthalten ist, welches bei neutraler Reaktion die Spaltung des Salicins in Saligenin und Zucker bewirkt. Ob dieses Ferment mit Emulsin identisch ist, ob es sich um ein spezifisches salicinspaltendes Ferment, Salikase, handelt, welches Wilhelm Sigmund ${ }^{1}$ ) zuerst in einigen Salix- und Populusarten aufgefunden hat, das müssen weitere Untersuchungen entscheiden.

\section{Resümee.}

In den frischen Bambusschößlingen lassen sich folgende Fermente nachweisen.

1. Nuclease.

2. Desamidasen, welche Harnstoff unter Bildung von Ammoniak zersetzen. Sie greifen auch das

1) W. Sigmund, Malys Jahresbericht, Bd. 38, S. 865. 
Asparagin an, aber in geringerem Maße als den Harnstoff. Das Glykokoll scheint durch diese Fermente nicht beeinflußt zu werden.

3. Ein fibrinlösendes Ferment. Über die Natur dieses Fermentes herrscht noch völlige Dunkelheit.

4. Diastasen, deren Wirkung in der Hydrolysierung der Stärke besteht. Als Endprodukt tritt Traubenzucker auf.

5. Ein emulsinähnliches Ferment, welches das Amygdalin in Benzaldehyd, Blausäure und Zucker zu spalten vermag.

6. Ein Ferment, welches befähigt ist, das Salicin in Saligenin und Zucker zu zerlegen. 